\title{
Belastungs- und Ressourcensituation operativer Führungskräfte bei virtueller Teamarbeit. Herausforderungen für die Gestaltung der Arbeit
}

\author{
Erich Latniak und Jennifer Schäfer
}

\subsection{Problemstellung}

Zunehmende Digitalisierung und vernetzte Kommunikationsinfrastrukturen haben für viele Unternehmen die Voraussetzungen geschaffen, um sog. virtuelle Teamarbeit zu nutzen: Beschäftigte arbeiten dabei an unterschiedlichen geografischen Orten in einem Team an einer gemeinsamen Aufgabe oder Problemlösung zusammen, und setzen dafür IT-gestützte Kommunikationsmedien in variabler Form und Intensität ein (vgl. Boos et al. 2017; Kauffeld et al. 2016, S. 44 f.; Gilson et al. 2015, S. 1317). In den letzten Jahren haben Videokonferenzsysteme und Plattformen, die mobile Endgeräte für den Austausch unterschiedlicher Datentypen einbinden können, weite Verbreitung gefunden (vgl. Roth und Müller 2017). Dies wirkt sich auf die Tätigkeiten der Teams aus. Die Unternehmen nutzen das zeit- und ortsunabhängige Arbeiten, um weltweit Kompetenzen zu erschließen, Kosten zu reduzieren und interne Abläufe zu beschleunigen (vgl. u. a. Manager Monitor 2017; PAC Group 2015; SHRM 2012).

Zur aktuellen Verbreitung dieser Arbeitsform in Deutschland gibt es unseres Wissens nach keine repräsentativen Untersuchungen, die verfügbaren Daten geben insofern eher grobe Orientierungen: Akin und Rumpf (2013, S. 377 f.) ermittelten bei einer Führungskräftebefragung, dass $75 \%$ der Unternehmen (mit Hauptsitz in Deutschland) bzw. $81 \%$ (mit Hauptsitz im Ausland) virtuelle Teams nutzten. Ähnliche Werte ermittelte auch der

E. Latniak $(\bowtie) \cdot$ J. Schäfer

Institut Arbeit und Qualifikation (IAQ), Universität Duisburg-Essen, Duisburg, Deutschland

E-Mail: erich.latniak@uni-due.de

J. Schäfer

E-Mail: jennifer.schaefer@uni-due.de

(C) Der/die Autor(en) 2021

S. Mütze-Niewöhner et al. (Hrsg.), Projekt- und Teamarbeit in der digitalisierten

Arbeitswelt, https://doi.org/10.1007/978-3-662-62231-5_4 
Manager Monitor (2017), zudem habe die Vertrautheit mit dieser Arbeitsform in den letzten Jahren bei den Befragten weiter zugenommen, was auf eine intensivere Nutzung hindeute. Virtuelle Teamarbeit hat sich offensichtlich zu einer in vielen Unternehmen genutzten, alltäglichen Form von Arbeit entwickelt (vgl. PAC Group 2015). Es ist anzunehmen, dass es, getrieben durch die fortschreitende Digitalisierung und Tertiarisierung, aber auch im Nachgang der aktuellen Corona-Krise sowie durch die arbeitspolitische Diskussion über ein Recht auf Homeoffice-Nutzung zur weiteren Verbreitung virtueller Team- und Projektarbeit kommen dürfte.

Diese Arbeitsform ist durch spezifische Herausforderungen gekennzeichnet, die sich als Anforderungen und Arbeitsbedingungen für die Beschäftigten niederschlagen. Hierzu gibt es zwar mittlerweile Forschungsbefunde in unterschiedlichen wissenschaftlichen Disziplinen (u. a. Kordsmeyer et al. 2019; Gilson et al. 2015; Malhotra und Majchrzak 2014; Maynard et al. 2012) ${ }^{1}$, insbesondere die Arbeitssituation der operativen Führungskräfte (opFk) in virtuellen Teams erweist sich allerdings als Forschungsdesiderat: Empirische Untersuchungen zur Arbeitssituation der opFk im deutschsprachigen Raum fehlen, soweit uns bekannt, bisher gänzlich für virtuelle Teamarbeit (vgl. Gilson et al. 2015, S. 1330; Syrek et al. 2013). Als opFk werden im Folgenden Team- und Projektleitende bezeichnet, wobei Teamleitende - je nach Organisation - disziplinarisch und fachlich für ,ihr' Team verantwortlich sind, während Projektleitende ihr(e) Projekt(e) zeitlich befristet leiten. In der bisherigen Forschung wird den opFk eine zentrale Rolle für Erfolg und Leistungsfähigkeit, aber auch für die Gesundheit der virtuell arbeitenden Teams zugeschrieben (vgl. Boos et al. 2017; Antoni und Syrek 2017; Hoegl und Muethel 2016, S. 8; Hoch und Kozlowski 2014; Akin und Rumpf 2013, S. 379).

Gegenstand in diesem Teilprojekt des vLead-Forschungsverbunds ${ }^{2}$ (vgl. die Beiträge von Antoni et al. und Hellert et al. in diesem Band) ist es vor diesem Hintergrund, für virtuell arbeitende opFk - exemplarisch in den Bereichen IT-Services bzw. Softwareentwicklung - zu untersuchen, wie deren Arbeitsbedingungen aussehen. Die IT-Branche bietet sich für ein exploratives Vorgehen besonders an, weil sie in der Nutzung der technischen Infrastruktur für verteiltes Arbeiten weit fortgeschritten ist (vgl. Roth und Müller 2017, S. 49 ff.).

Ziel des Teilprojekts ist es, Arbeitsbedingungen (Rahmenbedingungen), Belastungen und Ressourcen (vgl. Abschn. 3.1) der opFk explorativ zu untersuchen, um darauf

\footnotetext{
${ }^{1}$ Die Gegenüberstellung von ,lokalen` und ,virtuellen` Teams als distinkten Arbeitsformen gilt mittlerweile als überholt (vgl. u. a. Bailey et al. 2012; Will-Zocholl und Flecker 2019).

${ }^{2}$ Das Verbundprojekt „Modelle ressourcenorientierter und effektiver Führung digitaler Projektund Teamarbeit (vLead)“, in dessen Rahmen das Teilprojekt „Ressourcenstärkende Führung operative Führungskräfte in virtuellen Kontexten stärken und gesund erhalten“ vom 01.04.2017 31.12.2020 durchgeführt wird, wird gefördert vom Bundesministerium für Bildung und Forschung (BMBF) und dem Europäischen Sozialfonds unter dem Förderkennzeichen 2L15A081.
} 
aufbauend angepasste Maßnahmen bzw. Bausteine für ein präventives Ressourcenmanagement zu entwickeln, die die Leistungsfähigkeit und Gesundheit dieser Führungskräfte fördern bzw. erhalten sollen. Praktisches Ziel war es dabei, deren individuelle Handlungsfähigkeit durch Reflexion sowie durch besseres individuelles Ressourcenmanagement $\mathrm{zu}$ verbessern, wodurch sowohl der Erhalt der Arbeits- und Leistungsfähigkeit als auch die Effizienz und Effektivität der Arbeit unterstützt werden sollen. Dafür wurde u. a. ein leicht handhabbarer Fragebogen auf Screening-Niveau entworfen, der bei sog. Feedbackgesprächen mit den opFk eingesetzt wurde. Aufbauend auf den dabei gewonnenen Informationen wurden in diesen Gesprächen konkrete Vorschläge für Maßnahmen im Arbeitsumfeld oder bei der individuellen Arbeitsweise der opFk erarbeitet. Erste Ergebnisse, die im Rahmen der Gespräche mit dem Fragebogen erhoben wurden, werden im Folgenden dargestellt. Dabei stehen in diesem Beitrag drei Fragen im Vordergrund:

1. Welche spezifischen Herausforderungen stellen sich für operative Führungskräfte bei der Führung virtueller Teams bzw. der Arbeit in virtuellen Umgebungen?

2. Wie ist die Ressourcen- und Belastungssituation der opFk und wie unterscheidet sich diese zwischen höher und weniger beanspruchten opFk?

3. Welche Ansatzpunkte gibt es dabei für Verbesserungen der individuellen Arbeitssituation?

Im Weiteren werden zunächst die spezifischen Anforderungen an operative Führungskräfte in virtuellen Arbeitsumgebungen skizziert. Aufbauend darauf werden erste Befunde zur Ressourcen- und Belastungssituation der befragten opFk dargestellt. Aus diesen ersten Befunden werden schließlich Ansatzpunkte für Ressourcenmanagement und Arbeitsgestaltung abgeleitet.

\subsection{Aktuelle Herausforderungen der operativen Führungsarbeit in virtuellen Teams}

Für die operativen Führungskräfte ist charakteristisch, dass sie an einer multiplen Schnittstelle tätig sind und zwischen den unterschiedlichen Perspektiven und Interessen der internen sowie externen Kunden, dem strategischen Management (z. B. bei Ressourcenallokation bzw. Budgetfragen) und den jeweils ausführenden Teams vermitteln müssen (vgl. Latniak 2017). Ihre Tätigkeit lässt sich deshalb als kommunikationsintensives und spannungsreiches, Widerspruchsmanagement im Alltag' charakterisieren: OpFk kommt in virtuellen Teams die Aufgabe zu, die teilweise widersprüchlichen Vorgaben, Bedingungen und Ziele vereinbar zu machen (,Scharnierposition') und dabei überwiegend digital vermittelte Kommunikationsformen zu nutzen. Die konkreten Aufgabenstellungen variieren dabei erheblich; es gibt kaum einheitliche 
Aufgabenstrukturen, und auch die inhaltlich-fachliche Ausrichtung der Tätigkeiten ist ausdifferenziert.

Dabei mehren sich die Hinweise darauf, dass diese Konstellation zumindest bei einem relevanten Teil der opFk zu kritischen Beanspruchungssituationen beiträgt. So ermittelten u. a. Pangert und Schüpbach (2011), dass in den unteren Führungsebenen die Ressourcen geringer, die Stressoren stärker ausgeprägt waren als bei anderen Beschäftigtengruppen. Unterschiede fanden sich ebenfalls bei den Beanspruchungswerten, die bei den unteren Führungskräften höher ausfallen (a. a. O., S. 76). Dies wird durch Befunde von Zimber et al. (2015) sowie frühere eigene Untersuchungen bestärkt (Latniak 2017).

Vor welchen spezifischen Herausforderungen stehen die opFk in virtuellen Arbeitsumgebungen? Um die Anforderungen konkreter zu fassen wurden in einer Vorstudie auf Basis einer Literaturübersicht und ergänzender Interviews zur Arbeitssituation spezifische Herausforderungen herausgearbeitet, denen sich Team- und Projektleitende in virtuellen Teams gegenübersehen. Charakteristisch für die Arbeitssituation opFk in virtuell arbeitenden Teams sind auf dieser Basis die folgenden Aspekte:

1. Die opFk sind von der Motivation und Kooperationsbereitschaft der Teammitglieder für die Lösung gemeinsamer Aufgaben abhängig. Die Autonomie in der Arbeitsausführung und die Homeoffice-Nutzung erschweren es den opFk einerseits, Transparenz und Kontrolle über die jeweilige Arbeitsleistung der Teammitglieder zu gewinnen. Dies ist eine Quelle von Unsicherheit in der Kommunikation und in der Steuerung der Prozesse (vgl. Breuer et al. 2017). Der Team-Erfolg ist von der Leistungsbereitschaft der Teammitglieder abhängig (vgl. Boes und Kämpf 2019, S. 198), was die Steuerung und Kontrolle virtueller Teams anspruchsvoll macht, da auch bei Konflikten die Kooperation im Team gesichert werden muss. Gelingt die Integration der unterschiedlichen Perspektiven nicht, kann das zu psycho-sozialen Spannungen, emotionalen Belastungen und in der weiteren Bearbeitung zu Zusatzaufwänden beitragen. Im Gegensatz dazu können funktionierende Teams (durch Bereitstellen von Unterstützung) eine soziale Ressource darstellen.

2. In virtuellen Teams ist Personalführung tendenziell aufwendiger als dies bei traditioneller Führung der Fall ist. Einerseits schlägt sich der Vertrauensaufbau bei fehlenden direkten Kontakten häufig als Zusatzaufwand nieder (Breuer et al. 2017), etwa durch ergänzende Telefonate oder zusätzliche, reale" Treffen. Andererseits besteht bei Teammitgliedern, die ausschließlich im Homeoffice arbeiten und medienvermittelt kommunizieren, das Risiko in eine soziale Isolation zu geraten. Ob eine solche entsteht, ist in virtuellen Arbeitskontexten schwerer zu erkennen, und es muss dem bewusst entgegen gesteuert werden.

3. Durch die medienvermittelte Kommunikation liegt eine kontinuierliche Einbindung der opFk in parallele Informationsflüsse nahe (vgl. u. a. Seidler et al. 2018; Gilson et al. 2015, S. 1327), die in ein quasi ungeregeltes Multitasking führen kann. Einerseits sind störende Unterbrechungen, gerade bei der Bearbeitung konzentrations- 
intensiver Aufgaben, kaum vermeidbar, wenn parallel z. B. Chats genutzt werden. Gleichzeitig verkürzen sich die erwarteten Reaktionszeiten auf Anfragen; insgesamt ist von einer zunehmenden Dynamisierung der Abläufe auszugehen (vgl. insg. Korunka und Hoonakker 2014), die mit Zeitdruck oder mit Regulationshindernissen (wie z. B. Arbeitsunterbrechungen und informationsbezogener Mehraufwand, vgl. Ulich 2011, S. 127) einhergehen kann. Andererseits droht - bedingt durch die jeweiligen Arbeitszeiten bei weltweit verteilten Teams - eine zeitliche Ausdehnung der Arbeit in die Morgen- oder Abendstunden für notwendige Teamtreffen und Kommunikation. Damit verschwimmt die Abgrenzung zwischen Phasen der Arbeit und Phasen der Regeneration, die sich in einer Work-Life-,Imbalance“ niederschlagen kann.

4. Die IT-technischen Kompetenz-Anforderungen an opFk steigen, insbesondere wenn Groupware- oder Kollaborationssysteme neu eingeführt werden oder nicht gut in die technische Arbeitsumgebung integriert sind. Sie sind für die opFk zentrales Arbeitsmittel und müssen sicher beherrscht werden (vgl. den Beitrag von Hardwig et al. in diesem Band; zur Nutzung neuer Medien vgl. Gilson et al. 2015, S. 1323 ff.). Dies kann sich arbeitserleichternd auswirken; hier können aber auch Quellen für Regulationsbehinderungen (Ulich 2011, S. 126) liegen. In unseren Interviews erwies sich gerade die Möglichkeit zur Einarbeitung in neue Tools als eine kritische, weil knappe Größe. Neue Systeme und Kommunikationswege ersetzen dabei i. d. R. nicht die bereits vorhandenen Mittel, sondern ergänzen die Bisherigen: Diese parallele Nutzung mehrerer Kommunikationskanäle trägt zur Wahrnehmung von informationeller Überlastung bei, der sich die opFk ausgesetzt sehen (vgl. SchulzDadaczynski et al. 2019, S. 281; Drössler et al. 2018; Antoni und Ellwarth 2017; Eppler und Mengis 2004).

5. OpFk sind durch ihre Position in der Organisation in einer Doppelrolle als Geführte und Führende: Häufig werden sie selbst virtuell geführt. Damit fällt z. B. eine kurzfristige Rückmeldung durch die Vorgesetzten als mögliche Ressource aus. Unterstützung, von oben“ ist unter solchen Bedingungen nicht zeitnah verfügbar (vgl. Roth und Müller 2017). An dieser Stelle sind Rollen- und Zielklarheit sowie Unterstützung durch die eigenen Führungskräfte als Ressourcen (durch z. B. Feedback und Wertschätzung) bzw. - bei Fehlen derselben - als potenzieller Stressor zu berücksichtigen.

6. Meist ist die Position einer operativen Führungsaufgabe eine erste Bewährung für die weitere berufliche Entwicklung, die entsprechend gut bewältigt werden soll. Eine große Motivation der opFk für ihre Tätigkeit kann dazu beitragen, dass sie sich in die Tätigkeit auch mit großer Energie einbringen - oder in die Gefahr ,,interessierter Selbstgefährdung“ (Krause et al. 2015) geraten; gemeint ist damit ein Verhalten, bei dem Beschäftigte sich bewusst gesundheitsschädigend verhalten, um Misserfolge zu vermeiden. Dabei werden quasi die Leistungs- und Verhaltensmuster Selbstständiger von abhängig Beschäftigten übernommen.

Als weitere Herausforderung zeichnet sich für die opFk die zunehmende Verbreitung agiler Methoden der Softwareentwicklung und des Projektmanagements ab, die u. a. 
auch in einem der kooperierenden Unternehmen begonnen wurde. Mit den veränderten Rollen (wie z. B. Scrum Master oder Project Owner) und den anderen Ablaufstrukturen entstehen für die opFk neue Führungsanforderungen, die quer zum bisher im Unternehmen etablierten Führungshandeln liegen. Wie dies in virtuellen Arbeitskontexten in den Unternehmen umgesetzt werden kann und welche Bedingungen sich für die Beschäftigten und die opFk daraus ergeben ist eine offene Forschungsfrage (vgl. u. a. Boes und Kämpf 2019).

Diese Herausforderungen machen virtuelle Führung tendenziell zeit- und ressourcenintensiver als direkte Führung (vgl. Hoch und Kozlowski 2014), denn vieles, was in lokalen Teams in der direkten Interaktion emergent entsteht, muss in virtuellen Arbeitszusammenhängen bewusst hergestellt werden und führt $\mathrm{zu}$ zusätzlichen Arbeitsaufwänden. Gleichzeitig deutet sich anhand der skizzierten Spannungsverhältnisse an, dass die jeweils konkrete Ausprägung nicht ein-für-allemal gesetzt, sondern der Reflexion und Veränderung durch die Handelnden zugänglich ist. Insofern bietet sich hier durch Belastungsabbau bzw. ein entsprechendes Ressourcenmanagement die Möglichkeit, zu verbesserten Arbeitsbedingungen der opFk beizutragen.

\subsection{Elemente eines Ressourcenmanagements für operative Führungskräfte}

Vor diesem Hintergrund haben wir aufbauend auf den Befunden der Literaturanalyse und der Interviews mit opFk und Unternehmensvertretern relevante Ressourcen- und Belastungsfaktoren für den Screeningbogen ausgewählt und in den anschließenden Feedbackgesprächen erhoben. Die überarbeitete Endfassung des Instruments und die erarbeiteten Gestaltungsempfehlungen werden nach Projektende über die Webseite des Projekt verfügbar gemacht.

\subsubsection{Ressourcen- und Belastungskonzept}

Dabei verstehen wir Ressourcen als Handlungspotenziale, die von den Beschäftigten funktional unterstützend zur Bewältigung ihrer Anforderungen oder zur Reduzierung von Belastungen bzw. psychischer Kosten genutzt werden können (vgl. insg. Gerlmaier 2019a, S. 95 ff.). Wir gehen im Sinne des relationalen Belastungs- und Ressourcenkonzepts davon aus (vgl. Moldaschl 2005; Gerlmaier und Latniak 2007), dass Ressourcen nicht universell in allen Situationen als solche wirken, sondern erst im konkreten Gebrauch ihren Ressourcencharakter entfalten und insbesondere ihre stressreduzierende Wirkung von äußeren Bedingungen der Arbeitssituation bzw. der Handelnden abhängig sind.

Als mögliche situative Quellen gesundheitsförderlicher Ressourcen, die betrieblich beeinflusst werden können, unterscheiden wir im Anschluss an Gerlmaier (2019a) a) 
arbeitsorganisatorische und kapazitätsbezogene Ressourcen (kurz: ,Arbeitsressourcen'), die aus der Gestaltung des Arbeitssystems resultieren und z. B. in Form von Handlungsund Gestaltungsspielräumen, Kapazitätspuffern oder Arbeitstandems (vgl. Gerlmaier 2019c) stressreduzierend wirken können. ${ }^{3}$ Zweite wichtige Ressourcenquelle sind b) soziale Ressourcen, wie das soziale Klima im Team bzw. in der Organisation, soziale Unterstützung durch Kolleg*innen, oder ein unterstützendes Führungsverhalten des/r Vorgesetzten, für die jeweils stressmindernde Wirkungen als nachgewiesen gelten. c) Daneben gibt es Qualifikationsressourcen, die z. B. durch arbeitsimmanentes Lernen präventive Effekte in kritischen Situationen entfalten können. Hinzu kommen schließlich d) persönliche und Bewältigungsressourcen, die der Aufrechterhaltung der Gesundheit dienen können, wie etwa Selbstwirksamkeitserwartungen, Selbstmanagementfähigkeiten oder Erholungskompetenz. Sie wirken im Umgang mit den Anforderungen im Rahmen der organisatorisch vorgegebenen Möglichkeiten bei Nutzung problemlösend, belastungsreduzierend oder die Regeneration unterstützend.

Wir schließen uns darüber hinaus für die Entstehung psychischer Belastungen an das Konzept widersprüchlicher Arbeitsanforderungen an (vgl. Moldaschl 2005), das postuliert, dass psychische Belastungen in der Arbeit aus spezifischen Widersprüchen zwischen Anforderungen, Regeln und Ressourcen in der Arbeit entstehen, die von den Betroffenen dann nicht gelöst oder bearbeitet werden können (Einschränkung von Bewältigungsmöglichkeiten). Für den IT-Bereich wurden dafür 5 Widerspruchstypen identifiziert und operationalisiert (vgl. Gerlmaier und Latniak 2007, S. 135 ff.): Neben 1) widersprüchlichen Zielen in der Arbeit sind dies 2) Widersprüche zwischen Arbeitsanforderungen und Ausführungsbedingungen, 3) zwischen Aufgaben und Aneignungsbedingungen (Lernbehinderungen), 4) zwischen subjektiven Erwartungen und betrieblichen Zielen sowie 5) zwischen Arbeits- und Lebensweltanforderungen (WorkLife-Balance). Stress und Fehlbelastungen sind nach diesem Verständnis Resultat einer unzureichenden Ressourcensituation für die Bewältigung der Anforderungen bzw. Folge widersprüchlicher Konstellationen von Anforderungen, Regeln und Ressourcen in der Arbeit.

Die für den Screeningbogen ausgewählten Indikatoren genügen damit zwei Kriterien: Zum einen werden Indikatoren berücksichtigt, die im Rahmen der Vorstudie (Literaturauswertung, Interviews) als relevante Belastungs- und Ressourcenfaktoren identifiziert wurden. Hierzu zählten neben den skizzierten Spezifika virtueller Führung auch ,klassische' Regulationsbehinderungen wie z. B. Zusatzaufwand, Arbeitsunterbrechungen oder Zeitdruck, die oft durch individuelle Mehrarbeit kompensiert werden. Gleichzeitig sollte das Instrument relativ kurz bleiben, um ein zügiges Ausfüllen zu Beginn der Gespräche zu ermöglichen. (vgl. o., sowie Kordsmeyer et al. 2019). Zum anderen sind je mindestens eine Kurzskala bzw. ein Item der konzeptionell entwickelten

\footnotetext{
${ }^{3}$ Im Gegensatz zu Gerlmaier (a. a. O.) werden diese Ressourcentypen hier zusammengefasst, denn
} auch Kapazitätsressourcen sind aus unserer Sicht auch organisatorisch bedingt. 
Belastungs- und Ressourcentypen im Instrument berücksichtigt, um so ein möglichst breites Spektrum von Faktoren abzudecken. Auf Basis der Vorstudie (Literaturauswertung, Interviews) haben wir kapazitätsbedingte Aspekte (wie Zeitdruck, Work-LifeBalance), die informationsbezogenen Faktoren, Rollenklarheit und die Lerndimension berücksichtigt. Zum anderen standen individuelle Handlungspräferenzen im Fokus, die für die Feedbackgespräche gute Ansatzpunkte für unmittelbar umsetzbare Handlungsmöglichkeiten erschließen sollten.

\subsubsection{Instrument und Stichprobe}

Insgesamt haben wir für den Screeningbogen folgende Ressourcenaspekte übernommen (Tab. 4.1):

Für die Belastungen wurden folgende Aspekte berücksichtigt (Tab. 4.2):

Die Beantwortung erfolgte jeweils anhand angepasster 5-stufiger Vorgaben. Daneben wurden Fragen $\mathrm{zu}$ den individuellen und organisatorischen Rahmenbedingungen gestellt (u. a. zu Arbeitszeit und -orten, Zeitdifferenzen im Team, sowie Einschätzungen zu Teamkapazität/-stabilität/-kompetenzen) (vgl. Chudoba et al. 2005; Boos et al. 2017; Reif et al. 2018; Smith et al. 2011). Ergänzt wurde dies Output-seitig durch

Tab.4.1 Ausgewählte Ressourcen

\begin{tabular}{|c|c|c|}
\hline Тур & Items und Kurzskalen zu & Quellen \\
\hline $\begin{array}{l}\text { a) Arbeits- } \\
\text { organisatorische und } \\
\text { kapazitätsbezogene } \\
\text { Ressourcen }\end{array}$ & $\begin{array}{l}\text { - Rollenklarheit } \\
\text { - Gestaltungsspielraum } \\
\text { - Erholungsmöglichkeiten }\end{array}$ & \multirow{4}{*}{$\begin{array}{l}\text { In Anlehnung u. a. an } \\
\text { Gerlmaier 2011; Glaser et al. } \\
\text { 2015; Nübling et al. 2005; } \\
\text { Schnell 2018; Syrek et al., } \\
2011 \\
\text { ergänzt durch eigene Items im } \\
\text { Anschluss an Kaluza 2018; } \\
\text { Heinrichs et al. 2015; Reif } \\
\text { et al., 2018; Gerlmaier 2019a }\end{array}$} \\
\hline $\begin{array}{l}\text { b) Team- und soziale } \\
\text { Ressourcen }\end{array}$ & $\begin{array}{l}\text { - Unterstützung durch Führungskräfte } \\
\text { - Unterstützung durch Kolleg*innen } \\
\text { - Wertschätzung } \\
\text { - Feedback }\end{array}$ & \\
\hline $\begin{array}{l}\text { c) Qualifikations- } \\
\text { ressourcen }\end{array}$ & $\begin{array}{l}\text { - Möglichkeiten das eigene Wissen } \\
\text { zu erweitern }\end{array}$ & \\
\hline $\begin{array}{l}\text { d) persönliche und } \\
\text { Bewältigungsressourcen }\end{array}$ & $\begin{array}{l}\text { - Berufliche Sinnerfüllung } \\
\text { - Work-Life-Balance } \\
\text { - individuelles (instrumentales, } \\
\text { mentales und regeneratives) } \\
\text { Bewältigungsverhalten, z. B.: } \\
\text { - Nutzung von Kurzpausen } \\
\text { - Arbeiten nach der eigenen } \\
\text { Leistungskurve } \\
\text { - Situation mit Humor sehen } \\
\text { - Regeneration in der Freizeit z. B. } \\
\text { durch Sport }\end{array}$ & \\
\hline
\end{tabular}


Tab. 4.2 Ausgewählte Belastungen

\begin{tabular}{|c|c|c|}
\hline Widerspruchstyp & Items und Kurzskalen zu & Quellen \\
\hline $\begin{array}{l}\text { 1) widersprüchliche Ziele in } \\
\text { der Arbeit }\end{array}$ & • Rollenkonflikt & \multirow{5}{*}{$\begin{array}{l}\text { in Anlehnung u. a. an } \\
\text { Gerlmaier 2011; Nübling et al. } \\
\text { 2005; Bock et al. 2010; Böhm } \\
\text { et al. } 2017 \text { sowie Eigen- } \\
\text { kreationen }\end{array}$} \\
\hline $\begin{array}{l}\text { 2) Aufgabe vs. Ausführungs- } \\
\text { bedingungen }\end{array}$ & $\begin{array}{l}\text { - Arbeitsunterbrechungen } \\
\text { - Zusatzaufwand } \\
\text { - Zeitdruck } \\
\text { - Informationsüberflutung } \\
\text { - Kommunikationsrauschen } \\
\text { - Virtuelle Distanz }\end{array}$ & \\
\hline 3) Aneignung vs. Kapazität & - Aneignungsbehinderungen & \\
\hline $\begin{array}{l}\text { 4) widersprüchliche subjektive } \\
\text { Erwartungen und Ziele }\end{array}$ & $\begin{array}{l}\text { - psycho-soziale Belastungen/ } \\
\text { Emotionsarbeit }\end{array}$ & \\
\hline $\begin{array}{l}\text { 5) widersprüchliche } \\
\text { Anforderungen von Arbeits- } \\
\text { und Privatleben }\end{array}$ & - Entgrenzung & \\
\hline
\end{tabular}

Fragen zur arbeitsbedingten Motivierung, psychischem Erleben (Stress/Burnout) und Befindensbeeinträchtigungen als Beanspruchungsaspekten (u. a. Hacker und Rheinhold 1999; Mohr et al. 2005 in der Fassung von Gerlmaier 2011; Maslach und Jackson 1984 in der Fassung von Böhm et al. 2017; Fahrenberg 2004 in der Fassung von Gerlmaier 2011).

Eingesetzt wurde der Screeningbogen in der zweiten Jahreshälfte 2019 in insgesamt 24 Feedback-Gesprächen. Diese wurden überwiegend im direkten Kontakt geführt (23 Gespräche erfolgten face-to-face sowie eines video-basiert). Die Gespräche dauerten jeweils zwischen 90 und 120 min. Zu Beginn der Gespräche wurde der Screeningbogen von den Gesprächspartner*innen ausgefüllt.

Die Teilnehmenden kamen aus insgesamt 3 Unternehmen, davon ein großes ITService-Unternehmen, eine IT-Service-Tochterfirma eines großen produzierenden Konzerns, sowie einer Forschungseinrichtung. 23 Teilnehmende waren im IT- bzw. Softwareentwicklungsbereich tätig, ein/e Teilnehmende/r arbeitete in einem Forschungsbereich. Von den Teilnehmenden waren rund $71 \%$ männlich, $29 \%$ weiblich, etwa $55 \%$ hatten ein oder mehrere Kinder, die im Haushalt lebten. $50 \%$ der Befragten waren zum Befragungszeitpunkt zwischen 51 und 60 Jahren alt, 33,3\% zwischen 41 und 50, 12,5\% zwischen 31 und 40 Jahren, 4,2\% waren älter als 60 Jahre. $25 \%$ der Teilnehmenden verfügten über bis zu 5,5 Jahre, die anderen $75 \%$ bis zu 25 Jahre Führungserfahrung. Dabei waren rund $67 \%$ der Teilnehmenden disziplinarisch für ihre Teams verantwortlich; die Hälfte der Gesprächspartner*innen war (z. T. zusätzlich) projektleitend tätig, wobei diese Teilnehmenden zudem in der Regel mehr als ein Projekt leiteten. Die Mehrzahl der Befragten (ca. $71 \%$ ) hatte dabei mehr als 10 Mitarbeitende in ihrem wichtigsten Projekt. 


\subsection{Ergebnisse}

\subsubsection{Kontext der Befunde: Rahmenbedingungen und Arbeitssituation}

Virtuelle Teamarbeit ist für die befragten opFk weitgehend routinisiert und gelebter Alltag. Ihre Arbeit vollzieht sich primär digital vermittelt über Kollaborationsplattformen, Chats, E-Mail, Video- bzw. Telefonkonferenzen. Die Homeoffice-Nutzung wurde in einem IT-Dienstleistungsunternehmen (IT-Tochter eines großen Industriekonzerns, in dem wir die überwiegende Mehrzahl der Gespräche geführt haben) durch ein Desk Sharing-Konzept bei der Raumnutzung forciert; den Beschäftigten standen dort keine individuellen lokalen Büro-Arbeitsplätze mehr zur Verfügung. Es zeigte sich, dass sich ein Mix aus unterschiedlichen räumlichen Konstellationen eingespielt hat: Rund $71 \%$ der Befragten arbeiten selbst mindestens einmal wöchentlich vom Homeoffice (oder anderen Orten) aus.

Charakteristisch sind dort international zusammengesetzte Teams mit großen Zeitzonendifferenzen sowie kulturellen und sprachlichen Unterschieden. Mitarbeitende aus unterschiedlichen Unternehmensstandorten, von Dienstleistern sowie alternierend Telearbeitende sind eingebunden (,hybride“ Teams). Rund $75 \%$ der Befragten arbeiten dabei täglich mit Personen zusammen, die eine andere Sprache (als Muttersprache) sprechen, und rund $63 \%$ arbeiten täglich mit Beschäftigten aus unterschiedlichen Zeitzonen zusammen, was zu entsprechenden Restriktionen bei der Meeting-Planung beiträgt.

Die dafür notwendige technische Infrastruktur läuft aus Sicht der Befragten zufriedenstellend; lediglich individuelle technische Bedingungen einzelner Teammitglieder (z. B. instabile Leitung, mangelhaftes WLAN) führen hier zu Einschränkungen. Die Einschätzung der genutzten digitalen Medien durch die opFk ist überwiegend positiv, wobei Unterschiede hinsichtlich der digitalen Kommunikation im deutschen Sprachraum (83\%, gut/sehr gut'), über unterschiedliche Zeitzonen $(61 \%)$ und bei der Kommunikation mit Personen mit anderem sprachlichen oder kulturellen Hintergrund (54\%) festzustellen sind.

Das Arbeitsumfeld der Teilnehmenden ist hoch dynamisch: Es ist durch häufige Restrukturierungsmaßnahmen und Änderungen in der Teambesetzung gekennzeichnet. Die letzten 6 Arbeitsmonate der Teilnehmenden waren geprägt von Veränderungen in der Teambesetzung (ja: $88 \%$ der Teilnehmenden), Restrukturierungsmaßnahmen am Standort (75\%), Wechsel in den Arbeitsaufgaben/im Aufgabenfeld (46\%,) und häufig wechselnden Aufgabenprioritäten und Ziele bei den opFk und in ihrem Team (immer/oft: $50 \%$ ). Lediglich technisch verursachte Probleme traten vergleichsweise selten auf (25\% immer/oft).

Motivation und Kompetenz ihrer Teammitglieder für die anstehenden Aufgaben haben die Teilnehmenden als gut oder sehr gut bewertet (rund $67 \%$ bei der Kompetenz; $71 \%$ bei der Motivation). Problematischer wird hingegen die Personalkapazität für die 
anstehenden Aufgaben gesehen; hier gaben etwa $30 \%$ der Teilnehmenden an, dass diese , schlecht/sehr schlecht'sei.

Die Befragten arbeiten damit in einem hoch dynamischen organisatorischen Umfeld, das bis in die individuellen Aufgabenzuschnitte hinein häufigen Veränderungen unterworfen ist, und dies unter kritischen Kapazitätsvoraussetzungen bei etwa einem Drittel der Befragten.

\subsubsection{Ansatzpunkte für das Ressourcenmanagement}

Im Folgenden werden die explorativen deskriptiven Befunde $\mathrm{zu}$ den Belastungen von opFk und deren Nutzung von Arbeits- sowie Bewältigungsressourcen auf ItemEbene dargestellt. Im Anschluss daran wird untersucht, ob es erkennbare Unterschiede zwischen höher und weniger beanspruchten opFk gibt: Dafür wurde ein Mediansplit auf Grundlage der verwendeten Items/Skalen zu Beanspruchung (Stress/Burnout) durchgeführt (vgl. o.), um die Vergleichsgruppen zu bilden, die 11 (niedrigere Beanspruchungswerte) bzw. 13 Personen (mit höheren Werten) umfassten.

Betrachtet man zunächst die Angaben zu den psychischen Belastungen, so zeigt sich, dass 1) Unterbrechungen bei der Arbeit durch Personen und Telefonate und 2) das Erledigen beruflicher Dinge außerhalb der Arbeitszeit mit jeweils rund $71 \%$ am häufigsten (,trifft völlig/eher zu“) genannt werden, gefolgt von 3) Problemen an Weiterbildungsmaßnahmen teilzunehmen („Eine Teilnahme an beruflichen Weiterbildungsmaßnahmen gestaltet sich für mich schwierig") mit $54 \%$. Es folgt dann 4) der Zusatzaufwand bei der Beschaffung von Informationen, die vorliegen sollten (mit $50 \%)$, 5) die Erreichbarkeit für Personen, mit denen die opFk beruflich zu tun haben, auch außerhalb der Arbeitszeit (50\%), gefolgt von 6) großem Zeitdruck durch Terminvorgaben, 7) Unterbrechungen durch fehlende oder fehlerhafte Zuarbeiten, 8) der Notwendigkeit von Rückfragen bei unzulänglichen Informationen, 9) dem Gefühl, nicht alle Informationen effektiv verarbeiten zu können, sowie 10) der Aussage, dass Aufgaben eigentlich anders bearbeitet werden sollten und schließlich dem Item, dass 11) man sich während der Arbeit nicht angemessen in neue Sachverhalte einarbeiten kann (bei 6-11antworteten jeweils 37,5 \% aller Befragten ,trifft völlig/häufig zu“).

Dies verweist einerseits auf die große Bedeutung von Regulationshindernissen beim Belastungsgeschehen (5 Items u. a. zu Unterbrechungen und fehlenden Informationen), daneben werden die Probleme mit der Entgrenzung der Arbeit (mit 2 Items) sowie mit der Weiterbildung (2 Items) thematisiert, sowie Zeitdruck (als Regulationsüberforderung; vgl. Ulich 2011, S. 128 f.), Informationsverarbeitungsprobleme und kritische Bearbeitungsformen der jeweiligen Arbeitsaufgabe.

Für die Arbeitsressourcen ist bemerkenswert, dass die Befragten von einer für sie relativ günstigen Ressourcenausstattung berichten: Die höchsten Werte ermittelten wir für die Items 1) Verlässlichkeit von Kollegen, wenn es bei der Arbeit schwierig wird als 
ein Aspekt sozialer Unterstützung und 2) die Klarheit über den eigenen Verantwortungsbereich („Ich weiß genau welche Aufgaben in meinen Verantwortungsbereich fallen“) mit je 87,5\%, trifft völlig zu/eher zu' sowie 3) die Klarheit über die Erwartungen Anderer an die eigene Arbeit (mit rund $79 \%$ ). Deutlich wird auch die Bedeutung von beruflicher Sinnerfüllung (3 weitere Items unter den 10 am häufigsten genutzten) sowie der Work-Life-Balance (2 Items unter den 10 am häufigsten genutzten) im Erleben der Befragten. ${ }^{4}$

Die Gesprächspartner*innen nannten als am häufigsten genutzte Bewältigungsressourcen 1) die Erweiterung der Kompetenzen und des Wissens (79,2\% hier mit den Antwortkategorien ,immer/oft ${ }^{\circ}$ ), daneben 2) die verbesserte Planung und Organisation (70,8\%), sowie 3) Distanz schaffen zur Arbeit (66,7\%). Dieses Ergebnis ist insofern bemerkenswert als das am häufigsten genutzte Mittel (Wissen erweitern) gerade das ist, das im Alltag offenbar bei einigen der Befragten einem deutlichen Kapazitätsproblem unterliegt.

\subsubsection{Vergleich von operativen Führungskräften mit höherer und niedrigerer Beanspruchung}

\subsubsection{Belastungen}

Für den Mediansplit-basierten Vergleich von Gruppen unterschiedlich Beanspruchter zeigt sich, 1) dass deutliche Unterschiede beim erlebten Zeitdruck auftreten: Die höher Beanspruchten geben dabei zu fast $62 \%$ (,trifft völlig zu/eher zu“) an, dass sie nicht fertig werden, wenn sie ihre Arbeit gründlich erledigen wollen, während dies nur für rund $9 \%$ der geringer Beanspruchten gilt. Unterschiede zeigen sich auch 2) beim Aspekt ,Informationsüberflutung', bei dem rund $62 \%$ der höher Beanspruchten angaben (vs. $9 \%$ bei der Gruppe weniger Beanspruchter), nicht alle Informationen effektiv bearbeiten zu können. Rund $31 \%$ (vs. 18,2\%) gaben an, von den Informationen überwältigt zu sein. Auch das Item ,Ich verschwende viel Zeit damit, E-Mails und Sprachnachrichten zu beantworten, die (...) nicht direkt mit dem zusammenhängen, was ich erledigen muss“ belegt deutliche Unterschiede zwischen höher und weniger Beanspruchten (38,5\% vs. $9 \%$ ). Menge und Qualität der zu bearbeitenden Informationen scheinen damit bei den befragten operativen Führungskräften eine kritische Größe für Beanspruchungsrisiken darzustellen.

\footnotetext{
${ }^{4}$ Hohe Ausprägungen zur beruflichen Sinnerfüllung hatten „Meine Arbeit erfüllt mich“, „Meine beruflichen Tätigkeiten passen gut zu dem, was ich mir in meinem Leben vorgenommen habe“ und „Ich empfinde meine Arbeit als sinnvoll“ (79,2-70,8 \% „trifft völlig/eher zu“). Hohe Werte für Work-Life-Balance-Aspekte hatten ,Ich kann die Anforderungen aus meinem Privatleben und die Anforderungen aus meinem Berufsleben gleichermaßen gut erfüllen“ und „Es gelingt mir, einen guten Ausgleich zwischen belastenden und erholsamen Tätigkeiten in meinem Leben zu erreichen“ (75\% bzw, 70,8\% ,trifft völlig/eher zu“).
} 
Das Ausmaß der Beeinträchtigung von Tätigkeiten durch Arbeitsunterbrechungen unterscheidet 3) die beiden untersuchten Gruppen ebenfalls: Während fast $85 \%$ der höher Beanspruchten mit ,trifft völlig zu/eher zu“ bei dieser Frage antworteten, waren es unter den weniger Beanspruchten lediglich 54,5\%. Alle weiteren Fragen zum Zusatzaufwand ergaben keine großen Unterschiede zwischen den verglichenen Gruppen.

Diese zeigen sich schließlich 4) hinsichtlich der Aneignungsbehinderungen. Hier ergibt der Mediansplit allerdings ein widersprüchliches Bild: Einerseits geben fast $54 \%$ der höher Beanspruchten (vs. 18,2 \%) an, dass sie Probleme hätten, sich während der Arbeit angemessen in neue Sachverhalte einzuarbeiten. Andererseits bemängeln $73 \%$ der geringer Beanspruchten (vs. 38,5 \% der höher Beanspruchten), dass Teilnahmen an beruflichen Weiterbildungsmaßnahmen für sie schwierig wären. Für die Aneignungsbehinderungen zeigen sich damit möglicherweise zwei unterschiedliche Muster: Einerseits besteht bei den höher Beanspruchten die Tendenz, im Alltag keine Zeit mehr für die Einarbeitung in neue Themen zu finden, andererseits sind die weniger Beanspruchten - parallel dazu - nicht in der Lage, sich für Weiterbildungsmaßnahmen aus dem Alltag auszuklinken. Beide Muster deuten auf unterschiedliche Weise darauf hin, dass für die Befragten aus ihrer Sicht zu wenig Zeit oder Priorität im Alltag für Einarbeitung und Weiterbildung und damit für den Aufbau der zentralen Ressource Kompetenz gegeben ist.

Entgegen der Erwartungen verläuft die Verteilung beim Entgrenzungsaspekt; hier zeigen sich höhere Werte für die Erreichbarkeit in der Freizeit bei den geringer Beanspruchten. Offensichtlich gelingt es diesen besser, mit der zeitlich flexibilisierten Kommunikation in einer für sie schonenderen Weise umzugehen; in den Gesprächen wurden hierfür sehr unterschiedliche Umgangsformen berichtet. Zudem wiesen mehrere Gesprächspartner*innen darauf hin, dass sie zwar prinzipiell oder im Notfall erreichbar sind, dies aber insbesondere von ihren Vorgesetzten faktisch kaum in Anspruch genommen werde.

Insgesamt zeigen sich bei den Belastungen deutliche Anhaltspunkte für eine hohe Gesamt-Workload bei den Befragten, die bei den höher Beanspruchten tendenziell mit höherem Zeitdruck, mehr ,Informationsüberflutung' und häufigeren Unterbrechungen einhergeht.

\subsubsection{Ressourcen in der Arbeit}

Bei den Arbeitsressourcen zeigen sich für viele Faktoren nur relativ geringe Differenzen zwischen den unterschiedenen Gruppen, mit meist günstigeren Ausprägungen für die weniger Beanspruchten. Größere Differenzen zeigen sich allerdings 1) bei der Zielklarheit als einem Aspekt von Rollenklarheit. Hier liegen die Häufigkeiten der Antworten für das Item „Es gibt klare Ziele für meine Arbeit“ mit ,trifft völlig/eher zu“ bei 46,2 \% für die höher Beanspruchten gegenüber $72,7 \%$ bei den geringer Beanspruchten. Die beiden anderen Fragen dieser Skala liefern auf hohem Niveau ebenfalls günstigere Werte für die geringer Beanspruchten [,wissen welche Aufgaben im Verantwortungsbereich 
liegen` (76,9\% vs. $100 \%)$, wissen, was von mir bei der Arbeit erwartet wird` $(69,9 \%$ vs. $90,9 \%)$ ].

Nennenswerte Unterschiede zeigen sich 2) bei der Ressource Gestaltungsspielraum: Hinsichtlich der Möglichkeit, Arbeitsaufgaben an das Team zu delegieren, sind die Unterschiede zwischen beiden Gruppen zwar noch vergleichsweise gering $(61,5 \%$ für höher Beanspruchte vs. 72,7 \%). Bei der Möglichkeit, Einfluss auf Fertigstellungstermine oder Arbeitsvolumen zu nehmen, trennt sich dies weiter (38,5\% vs. 54,5\%) und erreicht die größte Differenz (30,8 \% vs. 54,5\%) beim Item „Ich kann Arbeitsvolumen oder Terminvorgaben mit dem Vorgesetzten oder Kunden neu aushandeln, wenn der Arbeitsanfall zu groß wird“. Insgesamt sind die Gestaltungsmöglichkeiten der höher Beanspruchten geringer ausgeprägt.

Unterschiede zeigen sich auch bei der 3) Unterstützung durch Kollegen sowie bei der 4) Unterstützung durch Vorgesetzte. Die Unterschiede sind dabei für die Unterstützung unter Kollegen auf hohem Niveau deutlich (76,9\% höher Beanspruchte vs. $100 \%$ ), für die Vorgesetztenunterstützung etwas geringer; sie deuten aber auf unterschiedlich gute Unterstützungsstrukturen hin, die für die Kompensation bzw. das Puffern von alltäglichen Lasten genutzt werden können.

Bemerkenswert sind schließlich noch die Unterschiede zwischen den beiden Gruppen bei den Werten für 5) Work-Life-Balance und berufliche Sinnerfüllung, die wir abgefragt haben (vgl. FN 4). Dabei ist zum einen auffallend, dass es den geringer Beanspruchten besser gelingt, einen Ausgleich zwischen Privat- und Berufsleben herzustellen. Bei der Bewertung sind allerdings die höher Beanspruchten insgesamt zufriedener. Schließlich zeigen die Werte für 6) berufliche Sinnerfüllung im Vergleich nennenswerte Differenzen zwischen den beiden Gruppen: Die höher Beanspruchten haben dabei höher ausgeprägte Werte (84,6 \% vs. 63,3\% bzw. zu 54,5\% bei den beiden Items).

Angesichts dieser Befunde verdichtet sich der Eindruck, dass die Gruppe der höher Beanspruchten einerseits über geringere Rollenklarheit, aber vor allem deutlich geringere Verhandlungs- und Entscheidungsspielräume verfügt als ihre geringer beanspruchten Kolleg*innen. Die Unterstützung ist dabei insgesamt hoch, allerdings auch hier besser für die geringer Beanspruchten mit deutlichen Unterschieden ausgeprägt. Bei der beruflichen Sinnerfüllung gibt es klärungsbedürftige Bezüge zwischen beruflicher Sinnerfüllung und Beanspruchung: Die Befunde könnten sich als Anzeichen für eine interessierte Selbstgefährdung (Krause et al. 2015) deuten lassen, bei der hohe Motivationswerte mit vergleichsweise höheren Beanspruchungen einhergehen.

\subsubsection{Bewältigungsressourcen}

Für die Bewältigungsressourcen zeigen sich beim Vergleich der höher und geringer Beanspruchten für die folgenden sechs Aspekte deutlichere Unterschiede: Zunächst gibt es Unterschiede 1) bei die Nutzung von Kurzpausen (vgl. Latniak 2019) und das 2) Arbeiten nach der persönlichen Leistungskurve (Gerlmaier 2019b, S. 327): Für Kurzpausen stellten wir fest, dass die höher Beanspruchten diese zu 38,5\%, immer/oft ${ }^{*}$ nutzen, während die geringer Beanspruchten sie zu 72,7 \% nutzen. Nur 15,4\% der höher 
Beanspruchten achten bei der Bearbeitung der Aufgaben (immer/oft) auf ihre persönliche Leistungskurve, während dass bei den geringer Beanspruchten zu 45,5\% der Fall ist. Hier besteht offenbar noch erhebliches Potenzial.

Relevant sind auch die Unterschiede, die sich hinsichtlich eher arbeitsorganisatorischer Aspekte [Arbeit besser gestalten 3) bessere Planung/Organisation 4) und 5) Lernmöglichkeiten (Kompetenzen/Wissen erweitern)] auf Grundlage der Befunde eröffnen. So unterscheiden sich die beiden Gruppen hinsichtlich der Nutzung dieser drei Aspekte deutlich: Eine aktive Bearbeitung der Verbesserungsmöglichkeiten von Arbeit (zusammen mit Kollege*innen und Vorgesetzten) nutzen 38,5\% der höher Beanspruchten, aber 63,6\% der geringer Beanspruchten, für bessere Planung und Organisation sind die Werte $61,5 \%$ vs. $81,8 \%$, für das Wissen-Erweitern $69,2 \%$ vs. $90,9 \%$. In der Summe nutzen also die weniger Beanspruchten diese Mittel deutlich häufiger.

Ein weiterer Unterschied betrifft 6) das Item ,Distanz zur Arbeit schaffen ': Die höher Beanspruchten nutzen dieses eher kognitive Coping zu 53,8 \% ,immer/oft", die geringer Beanspruchten zu 81,8 \%. Korrespondierend damit ist, dass Letztere zu 72,7 \% , selten/ nie' Freizeitaktivitäten reduzieren, während dies die höher beanspruchte Gruppe zu lediglich 53,8\% macht.

Kaum nennenswerte Unterschiede ergaben sich bei den ebenfalls erfragten Freizeitaktivitäten: Dies ist auch vor dem Hintergrund eines umfassenden Sport- und Beratungsangebots zu sehen, das von den Teilnehmenden z. T. intensiv genutzt wird (bei der überwiegenden Mehrheit der Teilnehmenden bestand hierzu ein Unternehmensangebot am Standort).

\subsubsection{Grenzen des Instruments und der Befunde - weitere Forschungsbedarfe}

Für diese Ergebnisse sind folgende Einschränkungen zu berücksichtigen: 1) Wir haben das Screeninginstrument nicht als Experteninstrument zum wissenschaftlichen Nachweis von Wirkungszusammenhängen ausgelegt, sondern als Hilfsmittel für einen praktischen Einsatz (z. B. beim Coaching). Die dargestellten explorativen Ergebnisse auf Item-Basis geben insofern eher Hinweise für weitergehende Forschungen. Es besteht weiterer Forschungsbedarf hinsichtlich der Güte des Instruments, das noch anhand einer größeren Stichprobe eingehender evaluiert werden sollte. 2) Die zweite Einschränkung liegt darin begründet, dass bei den Gesprächen ausschließlich freiwillig Teilnehmende mitwirkten. Dies birgt das Risiko einer Verzerrung der Ergebnisse, da die Stichprobe ggf. nur Interessierte mit verfügbarer Kapazität umfasst, während akut Überlastete sich dafür erfahrungsgemäß eher keine Zeit nehmen. 


\subsection{Arbeitspolitische und arbeitsgestalterische Ansatzpunkte}

Anhand dieser Befunde wird deutlich, dass die befragten operativen Führungskräfte mit häufigen Aufgaben- und Kontextwechseln konfrontiert sind, die entsprechenden Koordinations(-zusatz-)aufwand mit sich bringen, und zudem häufig parallel mehrere Aufgaben oder Probleme zu bearbeiten haben (Multitasking bzw. Mehrstellenarbeit). Dies geschieht teilweise unter Zeitdruck und bei einem hohen Anteil von - durch Meetings oder Kommunikation - verplanter Zeit. Wichtige Ressourcen sind dabei offenbar der Gestaltungsspielraum, Rollenklarheit, die Unterstützung durch Führungskräfte und Kolleg*innen, sowie die ausgewogene Work-Life-Balance. Die wesentlichen psychischen Belastungsfaktoren sind neben dem Zeitdruck und den Arbeitsunterbrechungen in erster Linie die mit der Kommunikation verbundenen Informationsverarbeitungsprobleme.

Vor diesem Hintergrund können präventiv ausgerichtete Arbeitsgestaltungsansätze darauf gerichtet sein, zu einer ,Entzerrung' und zu einer nach eigenen Möglichkeiten und Bedarfen geplanten Arbeitstätigkeit beizutragen. Wir knüpfen damit an die Überlegungen in Gerlmaier und Latniak (2013) an, wo dies als „Defragmentierung“ bezeichnet wurde: Diese Maßnahmen sind primär darauf gerichtet, insgesamt weniger Aufgaben parallel zu bearbeiten (d.h. die Ausführung der Aufgaben möglichst sequenziell durchzuführen), um damit die individuelle Informationsverarbeitung zu verbessern und die Aufgabenbearbeitung kontrollierbarer und sicherer zu machen. Dies hat nicht nur gesundheitspräventive Effekte, sondern trägt auch zur Effektivierung und sicheren Bearbeitung der Prozesse bei.

Eine ganze Reihe angesprochener belastungsreduzierender bzw. ressourcenaufbauender Maßnahmen gehören dabei schon zum gängigen instrumentellen Handlungsund Maßnahmenspektrum, das operativen Führungskräften zur Verfügung steht und genutzt wird, wie z. B. das Delegieren und Priorisieren, frühzeitig Unterstützung zu organisieren oder zusätzliche Kapazitäten zu schaffen sowie unterschiedliche kognitive Bewältigungsmaßnahmen (wie z. B. Maßnahmen zur Distanzierung von der Arbeit). Zudem zeigten sich Ansatzpunkte zur Verbesserung z. B. für die Nutzung von Kurzpausen, für die Berücksichtigung der persönlichen Leistungskurve, für den Wechsel von konzentrationsintensiven Aufgaben mit Aufgaben, die weniger konzentrationsintensiv sind, oder für das Festlegen von Zeiten für ungestörtes Arbeiten (Blockzeiten). Diese unterschiedlichen Ansätze gilt es weiter zu fördern und z. B. in Coaching-Gesprächen bzw. in Trainings einzuführen und zur Nutzung zu empfehlen.

Neben diesen eher individuellen Aspekten und den eher kleinschrittigen Maßnahmen zur Effektivierung virtueller Besprechungen oder des Mailverkehrs sehen wir auf Unternehmensebene eine Reihe von Ansatzpunkten, die zur Belastungsreduzierung an der für die opFk verfügbaren Arbeitskapazität ansetzen können. Es handelt sich dabei um regulierende Maßnahmen, wie z. B. eine 1) Begrenzung der Zahl der Projekte (Abbau von Mehrstellenarbeit, Begrenzungen für das Multitasking) oder der Führungsspannen, 
für die die opFk jeweils verantwortlich sind (Team- bzw. Projektgröße). Dies könnte zu reduziertem Zeitdruck beitragen. Dieses Ziel kann auch 2) mit dem Einsatz von ,Führungstandems“ erreicht werden, bei dem jeweils Leiter*in plus Stellvertretende/r für das jeweilige Team oder Projekt benannt werden und als Tandem in wechselseitiger Abstimmung arbeiten. Dies würde die individuellen Spielräume der opFk vergrößern und zudem die Möglichkeit eröffnen, sich einfacher in emotional kritischen Situationen auszutauschen und die Spannungen im Gesprächskontext abzufangen und teilweise abzubauen. Zudem hätte es den Nebeneffekt einer verbesserten Urlaubs- bzw. Krankheitsvertretung für das Unternehmen.

Ein weiterer entlastender Ansatzpunkt ist die 3) Einführung spezifischer Kostenstellen für Lernaufwände im Unternehmen, auf die Lernzeiten gebucht werden können. Dies würde dazu beitragen, einerseits notwendige Lernaufwände weniger in die Regenerationsphasen außerhalb der Arbeitszeit abzudrängen, und andererseits zu verdeutlichen, dass das Unternehmen dieses Lernen als eine Investition in die Beschäftigten begreift, die es zu fördern gilt.

Daneben deuten die dargestellten Befunde darauf hin, dass sowohl in der Arbeit wie an der Grenze zwischen Arbeitszeit und Regenerationsphasen ein bewussterer Umgang mit der eigenen Arbeitskraft bei den opFk unterstützt werden sollte. Für die Regeneration in der Arbeit sind u. a. die o. g. 4) Kurzpausen förderlich. Für die Arbeitszeitgrenzen bleiben 5) z. B. die in den beteiligten Unternehmen bereits geltenden Regelungen zu Wochenendarbeit (Mailverbot bzw. keine Beantwortung von E-Mails am Wochenende) weiter zu schulen bzw. zu reflektieren und ggf. anzupassen. Daneben sollte in 6) Trainings oder im Rahmen von Coachings verstärkt daran gearbeitet werden, dass die opFk eine für sich jeweils tragfähige Abgrenzung von Arbeit und Regeneration entwickeln, die z. B. durch bestimmte Handlungsmuster (Rituale wie die Tasse Tee nach dem ,nach Hause kommen“) unterstützt wird. Da die opFk in der Regel keine festgelegten Wochenarbeitszeiten haben, gibt es bei Zeitregelungen allerdings kaum wirkliche Ansatzpunkte; Vieles bleibt von der Interaktion zwischen opFk und den jeweiligen Vorgesetzten abhängig. Umso wichtiger wären hier Schulungen oder Trainings im Rahmen der Führungskräfteentwicklung, die zu Verhältnis von Verausgabung und Entspannung Informationen vermitteln, d. h. Gestaltungskompetenz aufbauen. Dies könnte praktisch umgesetzt und durch ,Lerngruppen“ sowie seitens der Vorgesetzten praktisch unterstützt werden.

Angesichts der skizzierten dynamischen Organisationsstrukturen erscheint es allerdings insbesondere notwendig, für eine regelmäßige Reflexion der Arbeitssituation zu sorgen, bei der leistungsbeeinträchtigende Arbeitsbedingungen in den Blick genommen werden. Zudem wäre dies die Gelegenheit, bei der (Arbeits-)Gestaltungskompetenzen im Ansatz vermittelt werden könnten. Dies wäre ein erster Schritt zur dauerhaften Verminderung der Belastungen und dem kontinuierlichen Aufbau von Ressourcen der operativen Führungskräfte in virtuellen Arbeitskontexten. 


\section{Literatur}

Akin N, Rumpf J (2013) Führung virtueller Teams. Gruppendynamik Organisationsberatung 44:373-387. https://doi.org/10.1007/s11612-013-0228-9

Antoni CH, Ellwarth T (2017) Informationsüberlastung bei digitaler Zusammenarbeit - Ursachen, Folgen und Interventionsmöglichkeiten. Gruppe. Interaktion. Organisation. Z Angewandte Organisationspsychologie (GIO) 48(4):305-315. doi: https://doi.org/10.1007/s11612-0170392-4

Antoni CH, Syrek C (2017) Digitalisierung der Arbeit. Konsequenzen für Führung und Zusammenarbeit. Gruppe. Interaktion. Organisation. Z Angewandte Organisationspsychologie (GIO) 48(4):247-258. doi: https://doi.org/10.1007/s11612-017-0391-5

Bailey DE, Leonardi PM, Barley SR (2012) The lure of the virtual. Organ Sci 23(5):1485-1504. https://doi.org/10.1287/orsc.1110.0703

Bock GW, Mahmood M, Sharma S, Kang YJ (2010) The impact of information overload and contribution overload on continued usage of electronic knowledge repositories. J Organ Comput Electron Commer 20(3):257-278. https://doi.org/10.1080/10919392.2010.494530

Böhm SA, Baumgärtner MK, Breier C, Brzykcy AZ, Kaufmann F, Kreiner P et al (2017) Lebensqualität und Lebenszufriedenheit von Berufstätigen in der Bundesrepublik Deutschland: Ergebnisse einer repräsentativen Studie der Universität St Gallen. Universität St. Gallen, St. Gallen

Boes A, Kämpf T (2019) Wie nachhaltig sind agile Arbeitsformen? In: Badura B et al (Hrsg) Fehlzeiten-report 2019. Springer Nature, S 193-204. doi: https://doi.org/10.1007/978-3-662-590447_13

Boos M, Hardwig T, Riethmüller M (2017) Führung und Zusammenarbeit in verteilten Teams. Hogrefe, Göttingen

Breuer C, Hüffmeier J, Hertel G (2017) Vertrauen per Mausklick: Wie vertrauen in virtuellen Teams entstehen kann. PERSONALquarterly 02(17):10-16

Chudoba KM, Wynn E, Lu M, Watson-Manheim MB (2005) How virtual are we? Measuring virtuality and understanding its impact in a global organization. Inf Syst J, 15(4):279-306

Drössler S, Steputat A, Schubert M, Günther N, Staudte R, Kofahl M, Hegewald J, Seidler A (2018) Informationsüberflutung durch digitale Medien. Systematischer Review qualitativer Studien. Zbl Arbeitsmedizin, Arbeitsschutz und Ergonomie 68:77-88. https://doi.org/10.1007/ s40664-018-0267-8

Eppler MJ, Mengis J (2004) The concept of information overload: a review of literature from organization science, accounting, marketing, MIS, and related disciplines. Inf Soc 20:325-344. https://doi.org/10.1080/01972240490507974

Fahrenberg J (2004) Die Freiburger Beschwerdenliste (FBL). Form FBL-G und revidierte Form FBL-R. Handanweisung. Hogrefe, Göttingen

Gerlmaier A (2011) Stress und Burnout bei IT-Fachleuten - auf der Suche nach Ursachen. In: Latniak E, Gerlmaier A (Hrsg) Burnout in der IT-Branche. Ursachen und betriebliche Prävention. Kröning, Asanger, S 53-90

Gerlmaier A (2019a) Neue Gestaltungsoptionen oder Null-Puffer? Arbeitsgestaltungspotenziale bei Anlerntätigkeiten, qualifizierter Facharbeit und produktionsnaher Wissensarbeit aus Sicht von betrieblichen Gestaltern und Beschäftigten. In Gerlmaier \& Latniak, S 93-124. https://link. springer.com/chapter/10.1007/978-3-658-26154-2_6

Gerlmaier A (2019b) Blockzeiten für störungsfreies Arbeiten. In Gerlmaier \& Latniak, S 325-328. https://link.springer.com/chapter/10.1007/978-3-658-26154-2_21

Gerlmaier A (2019c) Arbeitstandems. Gerlmaier \& Latniak 2019:319-324. https://link.springer. com/chapter/10.1007/978-3-658-26154-2_20 
Gerlmaier A, Latniak E (2007) Zwischen Innovation und alltäglichem Kleinkrieg: Arbeits- und Lernbedingungen bei Projektarbeit im IT-Bereich. In: Moldaschl M (Hrsg) Verwertung immaterieller Ressourcen: Nachhaltigkeit von Unternehmensführung und Arbeit III. Hampp, München, S 131-170

Gerlmaier A, Latniak E (2013) Psychische Belastungen in der IT-Projektarbeit - betriebliche Ansatzpunkte der Gestaltung und ihre Grenzen. In: Junghanns G, Morschhäuser M (Hrsg) Immer schneller, immer mehr: psychische Belastung bei Wissens- und Dienstleistungsarbeit. Bundesanstalt für Arbeitsschutz und Arbeitsmedizin (BAuA). Springer VS, Wiesbaden, S 165193. doi: https://doi.org/10.1007/978-3-658-01445-2_8

Gerlmaier A, Latniak E (Hrsg) (2019) Handbuch psycho-soziale Gestaltung digitaler Produktionsarbeit. Gesundheitsressourcen stärken durch organisationale Gestaltungskompetenz. Springer Gabler, Wiesbaden. https://link.springer.com/book/10.1007\%2F978-3-658-26154-2

Gilson LL, Maynard MT, Young NCJ, Vartiainen M, Hakonen M (2015) Virtual teams research: 10 years, 10 themes and 10 opportunities. J Manage 41(5):1313-1337. https://doi. org/10.1177/0149206314559946

Glaser J, Seubert C, Hornung S, Herbig B (2015) The impact of learning demands, work-related resources, and job stressors on creative performance and health. J Pers Psychol 14(1):37-48. https://doi.org/10.1027/1866-5888/a000127

Hacker W, Reinhold S (1999) Beanspruchungsscreening bei Humandienstleistungen (BHDSystem). Swets Test Serv, Frankfurt a. M.

Heinrichs M, Stächele T, Domes G (2015) Stress und Stressbewältigung. Fortschritte der Psychotherapie, Bd 58. Hogrefe, Göttingen

Hoch JE, Kozlowski SWJ (2014) Leading Virtual Teams: Hierarchical Leadership, Structural Supports, and Shared Team Leadership. J Appl Psychol 99(3):390-403. https://doi.org/10.1037/ a0030264

Hoegl M, Muethel M (2016) Enabling shared leadership in virtual projekt teams: a practitioners' guide. Proj Manage J 47(1):7-12. https://doi.org/10.1002/pmj21564

Kaluza G (2018) Stressbewältigung. Trainingsmanual zur psychologischen Gesundheitsförderung, 4., korrigierte Aufl. Berlin, Springer (Psychotherapie). doi: https://doi.org/10.1007/978-3-662$55638-2$

Kauffeld S, Handke L, Straube J (2016) Verteilt und doch verbunden: Virtuelle Teamarbeit. Gruppe. Interaktion. Organisation. Z Angewandte Organisationspsychologie (GIO) 47:43-51. doi: https://doi.org/10.1007/s11612-016-0308-8

Kordsmeyer AC, Mette J, Harth V, Mache S (2019) Arbeitsbezogene Belastungsfaktoren und Ressourcen in der virtuellen Teamarbeit. Zbl Arbeitsmedizin, Arbeitsschutz Ergonomie 48(4):239-244. https://doi.org/10.1007/s40664-018-0317-2

Korunka C, Hoonakker P (2014) The future of ICT and quality of working life: challenges, benefits, and riss. In: Korunka C, Hoonakker P (Hrsg) The impact of ICT on quality of working life. Dordrecht, Springer, S 205-220. doi: https://doi.org/10.1007/978-94-017-8854-0_13

Krause A, Baeriswyl S, Berset M, Deci N, Dettmers J, Dorsemagen C, Meier W, Schraner S, Stetter B, Straub L (2015) Selbstgefährdung als Indikator für Mängel bei der Gestaltung mobilflexibler Arbeit. Wirtschaftspsychologie 4-2014(1-2015):49-59

Latniak E (2019) Kurzpausen. In: Gerlmaier \& Latniak. S 371-375. https://link.springer.com/chap ter/10.1007/978-3-658-26154-2_29

Latniak E (2017) Ressourcenstärkende Führung - operative Führungskräfte in virtuellen Kontexten. Gruppe. Interaktion. Organisation. Z Angewandte Organisationspsychologie (GIO) 48(4):263-271. doi: https://doi.org/10.1007/s11612-017-0389-Z 
Malhotra A, Majchrzak A (2014) Enhancing performance of geographically distrubuted teams through targeted use of information and communication technologies. Hum Relat 67(4):386411. https://doi.org/10.1177/0018726713495284

Manager Monitor (2017) Umfragen und Stimmungsbilder zu aktuellen Themen aus Steuerpolitik, Sozialpolitik, Arbeitspolitik, Europapolitik und Management. Ausgabe 01/2017 vom 07. Februar 2017. https://www.ula.de/wp-content/uploads/2017/02/20170208-manager-monitor. pdf. Zugegriffen: 4. Dez. 2019

Maslach C, Jackson SE (1984) Burnout in organizational settings. In: Oskamp S (Hrsg) Applied social psychology annual: applications in organizational settings, Bd 5. Sage, Beverly Hills, S $133-153$

Maynard MT, Mathieu JE, Rapp TL, Gilson LL (2012) Something(s) old and something(s) new: modeling drivers of global virtual team effectiveness. J Organ Behav 33:342-365. https://doi. org $/ 10.1002 /$ job. 1772

Mohr G, Rigotti T, Müller A (2005) Irritation - ein Instrument zur Erfassung psychischer Befindensbeeinträchtigungen im Arbeitskontext. Skalen- und Itemparameter aus 15 Studien. Z Arbeits-und Organisationspsychologie, 49(1):44-48

Moldaschl M (2005) Ressourcenorientierte Analyse von Belastung und Bewältigung. In: Moldaschl M (Hrsg) Immaterielle Ressourcen: Nachhaltigkeit von Unternehmensführung und Arbeit I. Hampp, München, S 243-280

Nübling M, Stößel U, Hasselhorn HM, Michaelis M, Hofmann F (2005) Methoden zur Erfassung psychischer Belastungen: Erprobung eines Messinstrumentes (COPSOQ). Wirtschaftsverlag NW, Dortmund

PAC Group (2015). Zusammenarbeit virtueller Teams in deutschen Unternehmen. Relevanz, Herausforderungen, Lösungsstrategien. Executive Summary. https://www.pac-online.com/ download/19446/157014. Zugegriffen: 4. Dez. 2019

Pangert B, Schüpbach H (2011). Arbeitsbedingungen und Gesundheit von Führungskräften auf mittlerer und unterer Hierarchieebene. In: Badura B, Ducki A, Schröder H, Macco K (Hrsg) Fehlzeiten-Report 2011. Führung und Gesundheit. Springer, Berlin, S 71-79

Reif JAM, Spieß E, Stadler P (2018) Stress bewältigen. In: Reif JAM, Spieß E, Stadler P (Hrsg) Effektiver Umgang mit Stress. Gesundheitsmanagement im Beruf. Berlin, Springer. https:// ebookcentral.proquest.com/lib/gbv/detail.action?docID $=5387288$

Roth I, Müller N (2017) Digitalisierung und Arbeitsqualität. Eine Sonderauswertung auf Basis des DGB-Index Gute Arbeit 2016 für den Dienstleistungssektor. ver.di (Hrsg) Oktoberdruck AG, Berlin. https://innovation-gute-arbeit.verdi.de/themen/digitale-arbeit/++co++36c61f80-46a7$11 \mathrm{e} 7-\mathrm{b} 7 \mathrm{f5}-52540066 \mathrm{e} 5 \mathrm{a} 9$

Schnell T (2018) Von Lebenssinn und Sinn in der Arbeit. Warum es sich bei beruflicher Sinnerfüllung nicht um ein nettes Extra handelt. In: Badura B, Ducki A, Schröder H, Klose J, Meyer M (Hrsg) Fehlzeiten-Report 2018. Springer, Berlin, S 11-21

Schulz-Dadaczynski A, Junghanns G, Lohmann-Haislah A (2019) Extensives und intensives Arbeitenin der digitalisierten Arbeitswelt - Verbreitung, gesundheitliche Risiken und mögliche Gegenstrategien. In: Badura B et al (Hrsg) Fehlzeiten-Report 2019. Springer Nature, S 267283. doi: https://doi.org/10.1007/978-3-662-59044-7_18

Seidler A, Steputat A, Drössler S, Schubert M, Günther N et al (2018) Determinanten und Auswirkungen von Informationsüberflutung am Arbeitsplatz. Ein systematischer Review. Zbl Arbeitsmedizin, Arbeitsschutz Ergonomie 68:12-26. https://doi.org/10.1007/s40664-017-0252-7

SHRM [Society for Human Resource Management] (2012) SHRM survey findings: virtual teams. July 13, 2012. https://blog.shrm.org/trends/shrm-survey-findings-virtual-teams. Zugegriffen: 4. Dez. 2019 
Smith DC, Bruyns M, Evans S (2011) A project manager's optimism and stress management and IT project success. Int J Managing Projects Bus 4(1):10-27. https://doi. org/10.1108/17538371111096863

Syrek C, Apostel E, Antoni CH (2013) Stress in highly demanding IT jobs. Transformational leadership moderates the impact of time pressure on exhaustion and work-life balance. J Occup Health Psychol 18(3):252-261. doi: https://doi.org/10.1037/a0033085

Syrek C, Bauer-Emmel C, Antoni CH, Klusemann J (2011) Entwicklung und Validierung der Trierer Kurzskala zur Messung von Work-Life Balance (TKS-WLB). Diagnostica 57(3):134145. https://doi.org/10.1026/0012-1924/a000044

Ulich E (2011) Arbeitspsychologie, 7. Aufl. vdf, Zürich

Will-Zocholl M, Flecker J (2019) Zur realen Virtualität von Arbeit. Raumbezüge digitalisierter Wissensarbeit. Arbeits- und Industriesoziologische Studien 12(1):36-54

Zimber A, Hentrich S, Bockhoff K, Wissing C, Petermann F (2015) Wie stark sind Führungskräfte psychisch gefährdet? Z Gesundheitspsychologie 23(3):123-140. https://doi.org/10.1026/09438149/a000143

Open Access Dieses Kapitel wird unter der Creative Commons Namensnennung 4.0 International Lizenz (http://creativecommons.org/licenses/by/4.0/deed.de) veröffentlicht, welche die Nutzung, Vervielfältigung, Bearbeitung, Verbreitung und Wiedergabe in jeglichem Medium und Format erlaubt, sofern Sie den/die ursprünglichen Autor(en) und die Quelle ordnungsgemäß nennen, einen Link zur Creative Commons Lizenz beifügen und angeben, ob Änderungen vorgenommen wurden.

Die in diesem Kapitel enthaltenen Bilder und sonstiges Drittmaterial unterliegen ebenfalls der genannten Creative Commons Lizenz, sofern sich aus der Abbildungslegende nichts anderes ergibt. Sofern das betreffende Material nicht unter der genannten Creative Commons Lizenz steht und die betreffende Handlung nicht nach gesetzlichen Vorschriften erlaubt ist, ist für die oben aufgeführten Weiterverwendungen des Materials die Einwilligung des jeweiligen Rechteinhabers einzuholen.

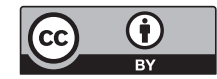

